\title{
CENTRAL LOCALIZATIONS OF REGULAR RINGS
}

\author{
E. P. ARMENDARIZ, JOE W. FISHER ${ }^{1}$ AND STUART A. STEINBERG
}

ABSTRACT. In this paper we show that a ring $R$ is von Neumann regular (or a $V$-ring) if and only if every central localization of $R$ at a maximal ideal of its center is von Neumann regular (or a $V$-ring). Strongly regular rings are characterized by the property that all central localizations at maximal ideals of the center are division rings. Also we consider whether regular PI-rings can be characterized by the property that all central localizations at maximal ideals of the center are simple.

Commutative von Neumann regular rings have been characterized in various ways. However, very few of these characterizations extend to noncommutative rings. The results in this paper arose from attempting to extend to noncommutative rings a well-known theorem of Kaplansky [11, Theorem 6] which states that commutative regular rings are characterized by the property that all localizations at maximal ideals are fields. It turns out that the obvious extension of this theorem to the noncommutative case is valid. That being, a ring is regular if and only if all central localizations at maximal ideals of the center are regular. An analogous theorem is obtained for $V$ rings. Also we show that strongly regular rings are characterized by the property that all central localizations at maximal ideals of the center are division rings.

With an eye to the commutative theory, we consider whether regular PIrings can be characterized by the property that all central localizations at maximal ideals of the center are simple. We provide an example to show that this is not the case. However, it is true if and only if contraction provides a 1:1 correspondence between maximal ideals of the ring and maximal ideals of the center.

Presented to the Society, January 17, 1974; received by the editors October 29, 1973. 16A38.

AMS (MOS) subject classifications (1970). Primary 16A30; Secondary 16A08,

Key words and phrases. Regular ring, strongly regular ring, V-ring, PI-ring, fully idempotent ring, central localization.

1 Research of the second author supported in part by NSF contract GP-38770. 
Throughout this paper $R$ will denote an associative ring which does have a unity and $C$ will denote the center of $R$. If $M$ is a prime ideal of $C$, then $C-M$ is a multiplicatively closed subset of $C$, and the ring of fractions of $R$ with respect to $C-M$ can be defined in the usual way. This ring of fractions is denoted by $R_{M}$ and is called the central localization of $R$ at M. As a $C$ module, $R_{M}$ is just the usual $C$-module localization of $R$. There is a canonical map $R \rightarrow R_{M}$ given by $r \rightarrow r / 1$.

A ring $R$ is called von Neumann regular (or just regular) if for each $a \in R$ there exists an $x \in R$ with $a=a x a$. If $R$ is a reduced ring, i.e., it has no nonzero nilpotent elements, and is also regular, then $R$ is called strongly regular. $R$ is called a $V$-ring if every simple right $R$-module is injective. By a PI-ring we mean a ring which satisfies a polynomial identity with coefficients in the centroid and at least one coefficient is invertible. If $R$ is a PIring, then $R_{M}$ satisfies the same PI as $R[12$, Lemma 1].

Lemma 1. Let $R$ be a ring with regular center $C$ and let $M$ be a maximal ideal of $C$. Then $M R=\operatorname{kernel}\left(R \rightarrow R_{M}\right), M R \cap C=M$, and $0 \rightarrow M R \rightarrow R \rightarrow$ $R M \rightarrow 0$ is exact.

Proof. First, image $\left(R \rightarrow R_{M}\right)=R_{M}$ because, if $c \in(C-M)$, then there exists $d \in C$ such that $(1-d c) c=0$ and $d \rightarrow 1 / c$. Let $A=\operatorname{kernel}\left(R \rightarrow R_{M}\right)$. Since $C$ is regular, $C_{M}$ is a field [11, Theorem 6], and hence $A \cap C=$ kernel $\left(C \rightarrow C_{M}\right)$ is a maximal ideal of $C$. Thus $A \cap C=M$ and so $M R \subseteq A$. If $a \in A$, then there exists $s \in(C-M)$ with $a s=0$. Because $C$ is regular, we have $(1-s t) s=0$ for some $t \in C$. Then $(1-s t) \in M$ and $a=(1-s t) a \epsilon$ $M R$. Therefore $A=M R$. This completes the proof.

A ring $R$ is fully (right) idempotent if $I^{2}=I$ for each (right) ideal $I$ of $R$. Regular rings are fully right idempotent but not conversely [4, Theorem 1.4], [6, Examples 3.1 and 3.2].

Proposition 2. A ring $R$ is fully (right) idempotent if and only if $R_{M}$ is fully (right) idempotent for each maximal ideal $M$ of $C$.

Proof. Let $l$ be an (right) ideal of $R$. Since $C_{M}$ is a flat $C$-module, $0 \rightarrow$ $\left(I^{2}\right)_{M} \rightarrow I_{M} \rightarrow\left(I / I^{2}\right)_{M} \rightarrow 0$ is exact and $I_{M}$ is an (right) ideal of $R_{M}$. Since $R_{M}$ is fully (right) idempotent, $I_{M}=I_{M}^{2}=\left(I^{2}\right)_{M}$. Thus $\left(I / I^{2}\right)_{M}=0$ for each maximal ideal $M$ of $C$. Therefore $I=I^{2}$ by $[3$, VII, Exercise 11$]$ and $R$ is fully (right) idempotent. Conversely, if $R$ is fully (right) idempotent, then according to [10, Lemma 2.3] $C$ is regular, and so image $\left(R \rightarrow R_{M}\right)=R_{M^{*}}$ Wherefore, $R_{M}$ is fully (right) idempotent. 
The next theorem is prompted by Kaplansky's conjecture that a semiprime ring is regular provided that all of its prime factor rings are regular [9, p. 2]. We obtain it as a corollary to Fisher and Snider's work on this conjecture [7].

Theorem 3. The following statements are equivalent for a ring $R$ with center $C$ :

(a) $R$ is regular.

(b) $C$ is regular and $R / M R$ is regular for each maximal ideal $M$ of $C$.

(c) $R_{M}$ is regular for each maximal ideal $M$ of $C$.

Proof. It is well known that the center of a regular ring is regular [15, Theorem 3], and hence (a) implies (b). Lemma 1 yields (b) implies (c). Suppose that each $R_{M}$ is regular. Then $R$ is fully idempotent by Proposition 2 and hence $C$ is regular. By Fisher and Snider [7, Corollary 1.3], a fully idempotent ring is regular exactly when each of its prime factor rings is regular. So let $P$ be a prime ideal of $R$. Then $P \cap C$ is a maximal ideal of $C$ and $(P \cap C) R \subseteq P$. From Lemma $1, R /(P \cap C) R \simeq R_{P \cap C}$ is regular. Wherefore $R / P$ is regular and (a) results. This completes the proof of the theorem.

Remark. The ring $R$ is called left $\pi$-regular if for each $a \in R$ there exists a positive integer $n$ and an $x \in R$ with $a^{n}=x a^{n+1}$. The following facts imply that $R$ is left $\pi$-regular if and only if $R_{M}$ is left $\pi$-regular for each maximal ideal $M$ of $C$.

(a) By $[2$, Lemma 1] $C$ is $\pi$-regular provided $R$ is left $\pi$-regular.

(b) As in Lemma 1 , if $C$ is $\pi$-regular, then $R \rightarrow R_{M}$ is onto and $\operatorname{kernel}\left(R \rightarrow R_{M}\right) \subseteq M R$.

(c) By [7, Theorem 2.1] $R$ is left $\pi$-regular provided all its prime factor rings are left $\pi$-regular.

Note that this adds another equivalence to Storrer [14, Lemma 5.6] and to Fisher-Snider [7, The orem 2.3], that being, in the former, a commutative ring $R$ is $\pi$-regular if and only if each $R_{M}$ is $\pi$-regular, and, in the latter, a PI-ring $R$ is $\pi$-regular exactly when each $R_{M}$ is $\pi$-regular.

A ring is biregular if each of its principal ideals is generated by a central idempotent. (If $R$ has no nonzero nilpotent elements, then it can be shown that $R$ is biregular if and only if $R_{M}$ is biregular for each maximal ideal $M$ of $C$.)

Lemma 4. The lattices of ideals of a biregular ring $R$, its center $C$, and its Boolean algebra of central idempotents $B$ are isomorphic via the mappings 


$$
\begin{array}{ll}
A \rightarrow A \cap C & (I R \leftarrow I), \\
A \rightarrow A \cap B & (J R \leftarrow J) .
\end{array}
$$

Proof. Since an ideal $A$ of $R$ is determined by its central idempotents, the mappings $A \rightarrow A \cap C$ and $A \rightarrow A \cap B$ are injective. Let $I$ be an ideal of $C$ and let $c \in(I R \cap C)$. Then $c=\Sigma c_{i} x_{i}, c_{i} \in I, x_{i} \in R$. Since $C$ is regular, $\Sigma C c_{i}=C e$ for some $e \in B \cap I$. Thus $c=c e \in I$ and $I=I R \cap C$. Whence $A \rightarrow A \cap C$ is surjective, and similarly $A \rightarrow A \cap B$ is surjective.

By extending the commutative property "all localizations at maximal ideals are fields" to "all central localizations at maximal ideals of the center are division rings," we obtain

Theorem 5. A ring $R$ is strongly regular if and only if $R_{M}$ is a division ring for each maximal ideal $M$ of $C$.

Proof. We first note that the ring $R$ is reduced exactly when each $R_{M}$ is reduced. For if $R$ is reduced and $(a / s)^{2}=0$ in some $R_{M}$, then $(a / 1)^{2}=0$ and so $a^{2} t=0$ for some $t \in C-M$. But then $(a t)^{2}=0$ implies $a t=0$, and so $a / s=0$. Thus $R_{M}$ is reduced. Conversely, if each $R_{M}$ is reduced and $a^{2}=0$, then $a / 1=0$ in each $R_{M}$. Whence $a=0$ and so $R$ is reduced. Using Theorem 3 we now have that $R$ is strongly regular if and only if each central localization $R_{M}$ is strongly regular. Also, if $R$ is strongly regular, then we have by Lemma 1 that $R_{M} \cong R / M R$ for each maximal ideal $M$ of $C$. Because $R$ is biregular we conclude, from Lemma 4 , that $R_{M}$ is a strongly regular simple ring, i.e., a division ring. This completes the proof.

By Kaplansky's theorem [11, Theorem 6] a commutative ring is a $V$-ring if and only if it is regular. In general, $V$-rings are not regular and vice-versa [4, Theorem 1.4], [6, Examples 3.1, 3.2 and 3.3]. It is true that $V$-rings are fully right idempotent [10, Corollary 2.2]. For an account of the relation between regular rings, $V$-rings, and fully right idempotent rings see Fisher [6].

Theorem 6. A ring $R$ is a $V$-ring if and only if $R_{M}$ is a $V$-ring for each maximal ideal $M$ of $C$.

Proof. If $R$ is a $V$-ring, then $C$ is regular [10, Lemma 2.3] and $R_{M}$ is a homomorphic image of $R$ by Lemma 1. Therefore $R_{M}$ is a $V$-ring. Conversely, if each $R_{M}$ is a $V$ ring, then each $R_{M}$ is fully right idempotent. Hence $R$ is fully right idempotent by Proposition 2, and $C$ is regular. But according to [6, Theorem 14] a fully right idempotent ring is a $V$-ring precisely when all its primitive factor rings are $V$-rings. Accordingly, let $P$ be a primitive 
ideal of $R$. Then $P \cap C$ is a maximal ideal of $C$ and $(P \cap C) R \subseteq P$. From Lemma $1, R /(P \cap C) R \simeq R_{P \cap C}$ is a $V$-ring. Wherefore $R / P$ is a $V$-ring.

Now we consider the question of whether regular PI-rings can be characterized as those for which all central localizations at maximal ideals of the center are simple. It follows immediately from Theorem 3 that a PI-ring $R$ is regular if $R_{M}$ is simple for each maximal ideal $M$ of $C$. The following example of a regular PI-ring $R$ with $R_{M}$ not simple shows that the converse is false.

Example. Let $R$ be the ring which consists of all sequences of $2 \times 2$ matrices with entries in a field $F$ which are eventually diagonal. Then $R$ is a regular PI-ring and $C$ is isomorphic to $\Pi_{i=1}^{\infty} F$. Let $M$ be a maximal ideal of $C$ which contains $\bigoplus_{i=1}^{\infty} F$. We claim that $R_{M}$ is not prime. Let $r_{1}=\left\{\left[\begin{array}{ll}1 & 0 \\ 0 & 0\end{array}\right]\right\}$ and $r_{2}=\left\{\left[\begin{array}{l}0 \\ 01\end{array}\right]\right\}$. Then $r_{1} 1^{-1} \neq 0$ and $r_{2} 1^{-1} \neq 0$, but an easy computation shows that $r_{1} 1^{-1} R_{M_{2}} 1^{-1}=0$. Consequently $R_{M}$ is not simple.

We can, however, characterize those regular PI-rings $R$ for which each $R_{M}$ is simple.

Theorem 7. The following statements are equivalent for a Pl-ring $R$ with center $C$.

1. $R_{M}$ is simple for each maximal ideal $M$ of $C$.

2. (a) $R$ is regular and

(b) the correspondence $M \rightarrow M R(I \cap C \leftarrow I)$ is a $1: 1$ correspondence between the maximal ideals of $C$ and the maximal ideals of $R$.

Proof. That (2) implies (1) follows immediately from Lemma 1. Suppose that (1) holds. Since $R_{M}$ is a simple PI-ring [12, Lemma 1], it is regular by a theorem of Kaplansky. Consequently $R$ is regular by Theorem 3. If $M$ is a maximal ideal of $C$, then $M R$ is a maximal ideal of $R$ by Lemma 1. Thus, if $I$ is a maximal ideal of $R$, then $I \cap C$ is a maximal ideal of $C$ and so $I=$ $(I \cap C) R$.

Corollary 8. Let $R$ be an Azumaya algebra which satisfies a PI. Then $R$ is regular if and only if $R_{M}$ is simple for each maximal ideal $M$ of $C$.

Proof. It follows from [5, Corollary 3.7, p. 54] that $M \rightarrow M R$ is a $1: 1$ correspondence between maximal ideals of $C$ and maximal ideals of $R$. Whence the result follows from Theorem 7.

Corollary 9. Let $R$ be a self-injective PI-ring. Then $R$ is regular if and only if $R_{M}$ is simple for each maximal ideal $M$ of $C$. 
Proof. Armendariz and Ste inberg [1, Theorem 3.5] show that a regular self-injective PI-ring is a finite direct sum of Azumaya algebras and hence is an Azumaya algebra. Thus an application of Corollary 8 and Theorem 7 completes the proof.

Corollary 10. If $R$ is a regular Pl-ring for which $\operatorname{Hom}_{R}(I, I)$ is a projective $R$-module for each ideal $I$ of $R$, then $R_{M}$ is simple for each maximal ideal $M$ of $C$.

Proof. By Steinberg [13, Corollary 8] $R$ is self-injective.

Corollary 11. If $R$ is a reduced PI-ring, then $R$ is regular if and only if $R_{M}$ is simple for each maximal ideal $M$ of $C$.

Proof. This follows from Theorems 5 and 7.

The Formanek center, $F(R)$, of a ring $R$ is the ideal of $C$ which consists of the values taken by all the Formanek central polynomials of $R[8],[12$, \$3]. An ideal of either $C$ or $R$ is called identity-faithful $[12, \$ 3]$ if it does not contain $F(R)$. Rowen [12, Theorem 3] shows that for a semiprime PI-ring $R$, the correspondence $M \rightarrow M R$ is a $1: 1$ correspondence between identityfaithful maximal ideals of $R$. Consequently, an application of Lemma 1 yields: If $R$ is a regular PI-ring, then $R_{M}$ is simple for each identity-faithful maximal ideal $M$ of $C$.

Note that the Example shows that even if $R$ is regular, $M \rightarrow M R$ need not be a $1: 1$ correspondence between all maximal ideals of $C$ and all maximal ideals of $R$, for this would contradict Theorem 7 .

\section{BIBLIOGRAPHY}

1. E. P. Armendariz and S. A. Steinberg, Regular self-injective rings with a polynomial identity, Trans. Amer. Math. Soc. 190 (1974), 417-425.

2. G. Azumaya, Strongly $\pi$-regular rings, J. Fac. Sci. Hokkaido Univ. Ser. I 13 (1954), 34-39. MR 16, 788.

3. H. Cartan and S. Eilenberg, Homological algebra, Princeton Univ. Press, Princeton, N. J., 1956. MR 17, 1040.

4. J. H. Cozzens, Homological properties of the ring of differential polynomials, Bull. Amer. Math. Soc. 76 (1970), 75-79. MR 41 \#3531.

5. F. Demeyer and E. Ingraham, Separable algebras over commutative rings, Lecture Notes in Math., vol. 181, Springer-Verlag, Berlin and New York, 1971.

6. J. W. Fisher, Von Neumann regular rings versus V-rings, Ring Theory: Proc. University of Oklahoma Conference, Dekker, New York, 1974, pp. 101-119.

7. J. W. Fisher and R. S. Snider, On the von Neumann regularity of rings with regular prime factor rings, Pacific J. Math. (to appear). 
8. E. Formanek, Central polynomials for matrix rings, J. Algebra 23 (1972), 129-132. MR $46 \# 1833$.

9. I. Kaplansky, Algebraic and analytic aspects of operator algebras, Regional Conf. Ser. in Math., vol. 1, Amer. Math. Soc., Providence, R. I., 1970.

10. G. Michler and $\mathrm{O}$. Villamayor, On rings whose simple modules are injective, J. Algebra 25 (1973), 185-201.

11. A. Rosenberg and D. Zelinsky, Finiteness of the injective hull, Math. Z. 70 (1958/59), 372-380. MR 21 \#4176.

12. L. Rowen, On rings with central polynomials, J. Algebra (to appear).

13. S. A. Steinberg, Rings of quotients of rings without nilpotent elements, Pacific J. Math. 46 (1973).

14. H. H. Storrer, Epimorphismen von kommutative ringen, Comment. Math. Helv. 43 (1968), 378-401. MR 39 \#4137.

15. J. von Neumann, On regular rings, Proc. Nat. Scad. Sci. U.S.A. 22 (1936), 707-713.

DE PARTMENT OF MATHEMATICS, UNIVERSITY OF TEXAS, AUSTIN, TEXAS 78712

DEPARTMENT OF MATHEMATICS, UNIVERSITY OF TOLEDO, TOLEDO, OHIO 43606 